\title{
Off-targets in RNAi screens
}

A systematic analysis of genome-wide short interfering RNA (siRNA) screens in human cells identifies widespread offtarget effects.

Gene knockdown by RNA interference (RNAi) is a powerful method for genetic screens in human cells. Nevertheless, this approach has many challenges. Perhaps foremost among them is the propensity of siRNAs to knock down the expression of genes other than the desired target. These off-target effects can occur when the siRNA is partially complementary to one or more cellular mRNAs (besides the target). The imperfectly matched oligonucleotide binds to these mRNAs and causes their perturbation, presumably via microRNA-like effects. Such off-target events, it has been previously shown, require matches between the mRNA and sequences that occur at 'seed' positions in the targeting oligonucleotide.

Researchers in multiple Swiss laboratories - the University of Zurich, University of Basel and ETH Zurich-now report a systematic study of seed-based offtarget effects in RNAi screens. Their analysis encompassed three independent genomewide screens aiming to identify host factors involved in infection of human cells by bacterial or viral pathogens. Each screen was conducted with the same commercially available siRNA library; for a subset of genes in the two bacterial infection screens, two additional libraries from different vendors were also used. All three libraries had about four siRNAs per gene.

The results of the analysis are striking and should give pause to any practitioner of RNAi screening in mammalian cells. Whereas there was almost no correlation in the examined phenotypes - cell survival and pathogen infection-caused by different siRNAs targeting the same gene, either within or between libraries, the phenotypic correlation was strong and statistically significant for siRNAs ostensibly targeting different genes but sharing a seed sequence. The implication is that the phenotypes in these three screens may largely be a consequence of seed-based off-target effects.

Indeed, when the researchers designed versions of infection-blocking oligonucleotides that still contained the seed sequence but lacked complementarity to the intended target gene, they observed that these siRNAs were as effective at blocking infection as the original versions. Mutations in the seed region, by contrast, abolished their activity.

RNAi screens notoriously produce many false positives, so researchers must sift through sometimes long lists of putative hits to identify genes that are genuinely involved in the phenotype they are studying. Widespread off-target effects, such as the ones observed here, may well be responsible for this phenomenon.

But what an investigator wants to find, after all, are the genes that cause a phenotype, irrespective of whether they do so via on- or off-target effects. Might there be information to be gleaned about causal genes also from siRNAs working through off-target effects? In principle, yes, says Christian von Mering at the University of Zurich, one of the lead authors on the study, but doing so in practice is not trivial. "This depends on predicting binding sites," he says. "And it's easy to do that, but in our experience, almost uninformative. [We] can predict two or three hundred binding sites for every given seed, but the ranking between these is almost random." In other words, identifying the true target genes of an siRNA with off-target effects can also require extensive secondary testing.

Redesign of siRNA libraries, von Mering explains, might help both to correct for offtarget effects and to make it easier to identify the underlying genes. If libraries were made up of oligos with only a few hundred seeds (many fewer than current libraries), each of them represented 20 or 30 times, the shift in the phenotypic distribution due to seedbased off-target effects could be more reliably learned, and corrected.

It remains to be seen whether RNAi will remain the dominant technology for genetic screens in human cells with the rise of haploid cell genetics and of the clustered, regularly interspaced, short palindromic repeats (CRISPR)-Cas9 system. But no method is perfect (off-target effects have been reported for the CRISPR system too). There is ample reason to understand the biases and limitations of all available methods as well as possible. Natalie de Souza

\section{RESEARCH PAPERS}

Franceschini, A. et al. Specific inhibition of diverse pathogens in human cells by synthetic microRNA-like oligonucleotides inferred from RNAi screens. Proc. Natl. Acad. Sci. USA 111, 4548-4553 (2014). 